\title{
X-ray spectroscopy of the cluster of galaxies PKS 0745-191 with XMM-Newton
}

\author{
Y. Chen ${ }^{1,2}$, Y. Ikebe ${ }^{2}$, and H. Böhringer ${ }^{2}$ \\ ${ }^{1}$ Center of Particle Astrophysics, Institute of High Energy Physics, Chinese Academy of Sciences, Beijing 100039, PR China \\ ${ }^{2}$ Max-Planck-Institut für Extraterrestrische Physik, 85740 Garching, Germany
}

Received 10 July 2002 / Accepted 4 June 2003

\begin{abstract}
We present the results from XMM-Newton observations of the cluster of galaxies PKS 0745-191. The spatially resolved spectra from the European Photon Imaging Cameras (both EPN and EMOS) can be fitted with a single temperature thermal plasma model when the interstellar column density is closer to the lower limit of the range allowed by observations. Alternatively, a two-component model can be fitted to the spectra from the inner region for slightly higher values of $N_{\mathrm{H}}$. The temperature profile shows a drop in the center of the cluster, from about $8 \mathrm{keV}$ at radii larger than $\sim 350 \mathrm{kpc}$ to about $4 \mathrm{keV}$ at the center, indicating that the gas in the central region is cooling. The total cooling flow rate inferred from the spectral data is only $364_{-94}^{+97} M_{\odot} \mathrm{yr}^{-1}$, however, much smaller than the previously obtained value. The metal abundance is found to decrease from 0.5 in solar units at the center to about 0.2 at a radius of $\sim 300 \mathrm{kpc}$. Deprojected spectra are also presented, which show a result similar to the projected spectra. The location of the X-ray emission peak is also the site of extended radio emission. Inspection of the higher resolution Chandra image of the cluster shows enhanced X-ray emission about 3' west of the center where also the radio emission is elongated. We explore possible implications on the interaction between the radio lobes and the intracluster plasma. Assuming that the gas is in hydrostatic equilibrium with the cluster potential, we find a total mass of about $1.18 \pm 0.04 \times 10^{13} M_{\odot}$ within the critical radius of the observed gravitational arc, which is a factor $\sim 2$ lower, however, than that from optical lensing. We also calculated the gas mass fraction profile up to $0.63 r_{\text {vir }}$ and obtained a mean gas mass fraction $f_{\text {gas }}=0.22 \pm 0.02$.
\end{abstract}

Key words. X-rays: galaxies - galaxies: clusters: individual: PKS 0745-191

\section{Introduction}

PKS 0745-191 is an X-ray luminous cluster of galaxies at redshift $z=0.1028$ (Fabian et al. 1985). Previous X-ray observations provided a global mean temperature of $8.5_{-0.8}^{+1.2} \mathrm{keV}$ (David et al. 1993). ROSAT data show that the cluster is elliptical and smooth, which implies that it is well relaxed (Allen et al. 1996). Moreover, the peak position of X-ray emission is approximately consistent with the optical Central Cluster Galaxy (CCG) coordinates (Allen et al. 1996). From ASCA, the central Intracluster Medium (ICM) is found to contain a massive cooling flow of $\sim 1000 M_{\odot} \mathrm{yr}^{-1}$ (Allen et al. 1996; Allen 2000). With combined GIS2 and GIS3 data, the gas temperature, determined by a single temperature model, is about $8.1_{-0.9}^{+1.0} \mathrm{keV}$ in the annular region $3^{\prime}-6^{\prime}$ from the center (Allen et al. 1996). Ikebe et al. (2002) measured a mean temperature of $6.4_{-0.2}^{+0.2} \mathrm{keV}$. Ikebe determined global temperatures with $2 \mathrm{~T}$ model (the $2 \mathrm{~T}$ also accounts for spatial variation), while Allen uses spatially resolved data but includes a cooling flow. The difference in the methods may well explain the different results. In addition, BeppoSAX showed a metal abundance

Send offprint requests to: Y. Chen, e-mail: ychen@mail.ihep.ac.cn decreasing from 0.4 in solar units in the center to 0.2 at radii larger than $350 \mathrm{kpc}$ (De Grandi \& Molendi 1999).

In the optical energy band, PKS 0745-191 is also known to have a bright strong lensing arc and two fainter arc candidates (Allen et al. 1996). The lensing mass $\left(\sim 2.5 \times 10^{13} M_{\odot}\right)$ is reported to be in agreement with $\mathrm{X}$-ray results, when a multiphase analysis is adopted (Allen et al. 1996). However, the mass determined from X-ray method of some other lensing clusters is found to be systematically lower than that from the strong gravitational lensing method by a factor $\sim 2$ (e.g. Loeb \& Mao 1994; Wu 1994; Miralda-Escudé \& Babul 1995). With Chandra data, the X-ray mass of some clusters is recently found to be consistent with the optical lensing mass (e.g. A2390, Allen et al. 2001; A1835, Schmidt et al. 2001). However, the discrepancy still exists in some other clusters (e.g. A2218, Machacek et al. 2002; A1689, Xue \& Wu 2002). This may be due to the fact that the gas is not in thermal hydrostatic equilibrium in the central region of the cluster or there is apparent offsets between X-ray and lensing centers (e.g. Allen 1998). Moreover, Loeb \& Mao (1994) suggest a nonthermal pressure support in the cluster core to explain the discrepancy.

PKS 0745-191 is also known as a high luminosity radio source. The Very Large Array (VLA) observations show that 
Table 1. Summary of XMM observations.

\begin{tabular}{cccccc}
\hline \hline Observations & ID & $\begin{array}{c}\text { Date } \\
\text { yyyy-mm-dd }\end{array}$ & $\begin{array}{c}\text { Duration } \\
(\mathrm{s})\end{array}$ & $\begin{array}{c}\text { Pointing RA } \\
(\mathrm{J} 2000)\end{array}$ & $\begin{array}{c}\text { Pointing Dec } \\
(\mathrm{J} 2000)\end{array}$ \\
\hline Off-set & 0105870201 & $2000-10-30$ & 44169 & $07^{\mathrm{h}} 48^{\mathrm{m}} 22^{\mathrm{s}} .9$ & $-19^{\circ} 04^{\prime} 26^{\prime \prime} 9$ \\
\hline Center & 0105870101 & $2000-10-31$ & 28369 & $07^{\mathrm{h}} 47^{\mathrm{m}} 28^{\mathrm{s}} .9$ & $-19^{\circ} 16^{\prime} 04^{\prime \prime} 3$ \\
\hline
\end{tabular}

the radio source contains a core as well as diffuse emission (Baum \& O'Dea 1991). Moreover, the pressure of X-ray emitting gases exceeds the minimum pressure in the radio emitting plasma by 1-2 orders of magnitude in the central region of the cluster (Baum \& O’Dea 1991; Sarazin et al. 1995). One way to explain this excess is that there is lots of energy in relativistic protons in the central region (Baum \& O'Dea 1991).

In a number of cooling clusters, the radio plasma displaces the X-ray emitting gas by radio jet and thus the radio and X-ray emitting regions anticorrelate (e.g. Perseus cluster, Böhringer et al. 1993; Hydra A, McNamara et al. 2000; A2597, McNamara et al. 2001; A478, Sun et al. 2003). While in the case of PKS 0745-191 and some other clusters (e.g. M 87, Böhringer et al. 1995; 2A 0335+096, Sarazin et al. 1995), the radio plasma moves outward buoyantly and may also entrain a lot of thermal gas (Sarazin et al. 1995). The entrained gas can enhance the X-ray emission and may form some mushroom like substructure in the inner region of the cluster (Churazov et al. 2001).

This paper reports the results of deep X-ray observations with XMM-Newton of the cluster PKS 0745-191. In Sect. 2, we describe the observations and the data preparation and in Sect. 3 we present the spectral analysis results from the XMM-Newton observations. In Sect. 4 we compare the X-ray and radio morphology of the inner region of the cluster. In Sect. 5 we determine the total projected mass within the critical radius and calculate the gas mass fraction. We provide discussions and a summary in Sect. 6. In this paper, we assume $H_{0}=50 \mathrm{~km} \mathrm{~s}^{-1} \mathrm{Mpc}^{-1}, \Omega_{m}=1$ and $\Omega_{\Lambda}=0$.

\section{Observations and data preparation}

Two observations of PKS 0745-191 were performed with XMM-Newton in October 2000, one is pointing near the center of the cluster, the other has an off-set of $18^{\prime}$ (151 kpc per arcmin) to the NE direction (see Table 1 and Fig. 1). With these two observations the cluster emission is covered in the NE direction well beyond the estimated virial radius of the cluster.

There are some periods with "flares" in both observations. The count rate in the last $\sim 6 \mathrm{ks}$ of the exposure time is slightly higher than that in the first $\sim 5 \mathrm{ks}$ and this will probably affect the spectral analysis, especially in the outer regions. Therefore, we only use the first $\sim 5 \mathrm{ks}$ for the analysis of the outer regions with $R>4$.'2 and both parts for the inner regions with $R<4$.'2. After removing those "flare" periods, we got a good exposure time of about $10.6 \mathrm{ks}$ for EPN (both parts) and $15.7 \mathrm{ks}$ for EMOS for the center observational data. The off-set data have $20.8 \mathrm{ks}$ good exposure time for both instruments.

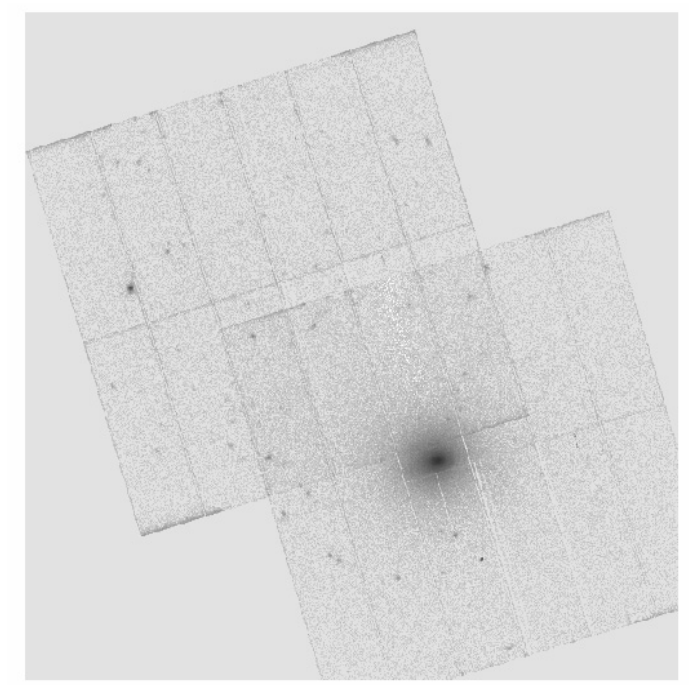

Fig. 1. The exposure and out of time events corrected EPN image of the two observations. The pixel size is $4^{\prime \prime} \times 4^{\prime \prime}$.

\subsection{Background subtraction}

PKS 0745-191 is located near the galactic plane and has a high galactic absorption with a value of $N_{\mathrm{H}}=4.24 \times 10^{21} \mathrm{~cm}^{-2}$ (e.g. compared to that of the Lockman hole with $N_{\mathrm{H}}=6 \times$ $10^{19} \mathrm{~cm}^{-2}$ ). Therefore, we can not use the Lockman hole data to determine the background. We use the off-set observational data from the same detector regions instead. Moreover, we can estimate the uncertainty of the $N_{\mathrm{H}}$ value from the coarse grained $\left(2^{\circ} \times 2^{\circ}\right) 21-\mathrm{cm}$ data (Dickey \& Lockman 1990) by inspecting the variation of $N_{\mathrm{H}}$ in the immediate surroundings of PKS 0745-191, where values are found ranging from 3.2 to $4.4 \times 10^{21} \mathrm{~cm}^{-2}$ (see Allen 2000). Due to this large uncertainty it is necessary to obtain the best value for $N_{\mathrm{H}}$ from fits to the X-ray data. The result is not unique for a variety of possible fitting models and we will discuss the most plausible value for this parameter.

The process of background subtraction can be divided into three steps. (1) We subtract the background determined by the off-set observational data. (2) We compare the spectra obtained from the corners of the detectors of these two observations. If there is excess particle background in the center observational data, we subtract it from the source spectra. Note that here we assume that this kind of background has no vignetting effect. Usually, this background contributes only a small portion to the total background. (3) After performing the above two steps, we found an additional residual background. Figure 2 (top panel) shows a typical spectrum which was obtained from the region with a radius $4.2<R<5.8$ and was fitted with an absorbed 

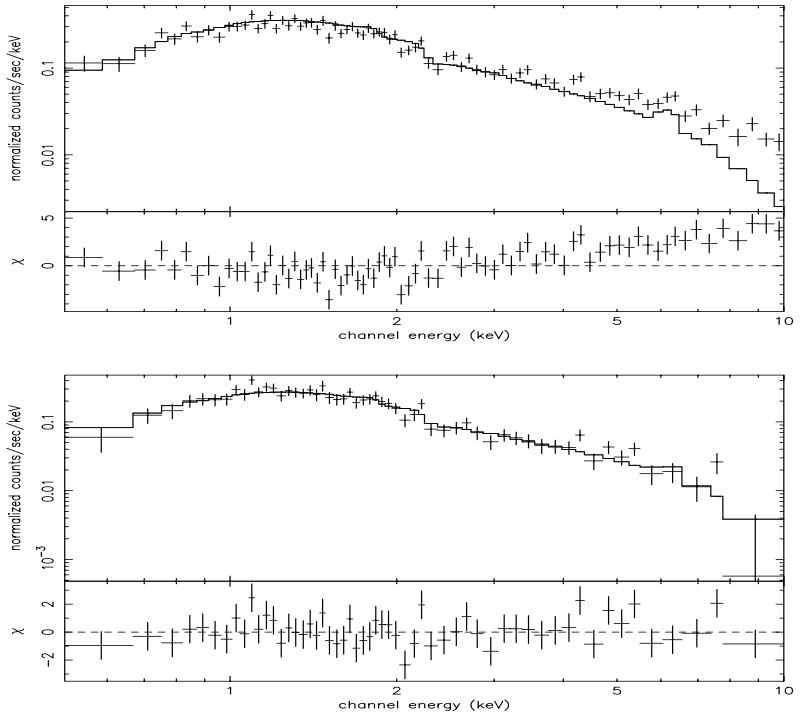

Fig. 2. Top panel: EPN spectrum of the 4!2-5!8 radial region in PKS 0745 without additional background correction. Bottom panel: Spectrum after correction of additional background.

thermal plasma Mekal model (Kaastra et al. 1996) with a fixed temperature $9.0 \mathrm{keV}$ in XSPEC (Arnaud 1996). Please note the large residues in the hard energy band. This background is due to the fact that the observing conditions of the off-set and the central pointing are not exactly the same and the background in the central pointing is slightly higher. This background may be mostly caused by low level proton flares imaged through the mirrors (Lumb 2001). We use events in an annular region far away from the center, with a radius $11.6<R<15^{\prime}$. 0 , to determine this kind of background. The quality of the spectrum improved significantly after the correction was applied (Fig. 2, bottom panel).

After these three steps of background subtraction, the spectra are now ready to be analyzed.

\section{Spectral analysis}

The image of the cluster is divided into 10 annular regions centered on the emission peak for the extraction of spectra (Fig. 3). Because there is significant cluster emission in the South-West region in the image of the off-set observation, this region should be excluded from the background determination. We also excluded the same detector region in the central observational image. We use the outermost region, with a radius 11 . $6<R<15$ '0, to determine the background in step 3. All spectral fits are performed in the $0.5-10 \mathrm{keV}$ band for both EPN and EMOS. For EPN, the single events (PATTERN = $0)$ spectrum and the double events $(1 \leq$ PATTERN $\leq 4)$ spectrum from each annular region are fitted simultaneously. While the spectra from EMOS1 and EMOS2 are also fitted simultaneously.

\subsection{Projected spectra}

As discussed in the preceding section, we first set $N_{\mathrm{H}}$ free and use the $0.25<R<4$.'2 region to obtain a best fit value of $N_{\mathrm{H}}$.

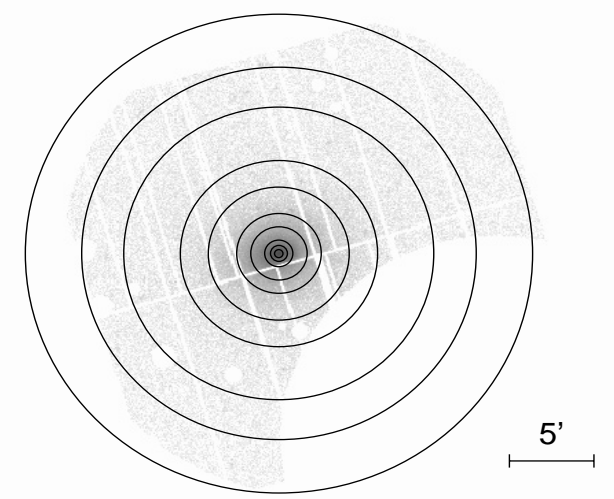

Fig. 3. Annular regions. The outermost annular region is used to determine the additional background. The SW corner is excluded because there is no corresponding uncontaminated background region in the off-set pointing used for the background subtraction.

We got $N_{\mathrm{H}}=3.6 \times 10^{21} \mathrm{~cm}^{-2}$ for the EPN spectra and $N_{\mathrm{H}}=$ $4.0 \times 10^{21} \mathrm{~cm}^{-2}$ for the EMOS spectra in good agreement with the $21 \mathrm{~cm}$ results. Then we fix $N_{\mathrm{H}}$ to those values and use some models to fit the spectra.

We fit the spatially resolved projected spectra from both EPN and EMOS with a single temperature Mekal model out to a radius about $9^{\prime}$ (also see Table 2 and Fig. 4a). Please note that the spectra from the outer region $(9.2<R<11$ ' 6 ) have a poor signal to noise ratio and are not listed in Table 2. The innermost region and regions with $R>4 . ' 2$ have relatively high $\chi^{2}$ values. For the innermost region, this may suggest the existence of another component. Therefore we explore in the following part of this section where we analyze the deprojected spectra, if the fit can be improved by a second temperature component. For the outer region, the high $\chi^{2}$ values may be due to high background and some possibly systematic errors caused by the background subtraction.

The radial temperature profile (Fig. 5) shows a drop in the center of the cluster, from about $8 \mathrm{keV}$ at radii larger than $\sim 350 \mathrm{kpc}$ to about $4 \mathrm{keV}$ in the innermost bin, indicating that the gas in the central region is cooling. The metal abundance is found to decrease with the radius from 0.5 in solar units at the center to about 0.2 at a radius of $\sim 450 \mathrm{kpc}$, which is consistent with the result from BeppoSAX (De Grandi \& Molendi 1999).

\subsection{Deprojected spectra}

Since the temperature varies among these annular regions, the projected spectra of the inner regions are contaminated by the emission of the outer regions. From these projected spectra, assuming spherical symmetry, we can derive deprojected spectra.

This time we fit the EPN spectra with the $N_{\mathrm{H}}$ value fixed to the galactic value, i.e. $N_{\mathrm{H}}=4.24 \times 10^{21} \mathrm{~cm}^{-2}$ first and then change it to the best fit value $3.6 \times 10^{21} \mathrm{~cm}^{-2}$. The results are shown in Table 3 (also see Figs. 4b, c). We found that the $\chi^{2}$ values are significantly decreased for EPN 
Table 2. Fit of projected spectra with a single temperature model. The $N_{\mathrm{H}}$ value is fixed to $3.6 \times 10^{21} \mathrm{~cm}^{-2}$ for EPN and $4.0 \times 10^{21} \mathrm{~cm}^{-2}$ for EMOS. Note that the errors in all of the spectral analysis in this paper represent 90 per cent $\left(\Delta \chi^{2}=2.71\right)$ confidence limits.

\begin{tabular}{|c|c|c|c|c|c|c|}
\hline Radius & $T(\mathrm{keV})$ & Abundance & $x^{2}$ & $T(\mathrm{keV})$ & Abundance & $\chi^{2}$ \\
\hline Instrument & & EPN & & & EMOS & \\
\hline $0^{\prime}-0.25$ & $4.4_{-0.2}^{+0.2}$ & $0.46_{-0.08}^{+0.09}$ & $135 / 98=1.37$ & $4.5_{-0.1}^{+0.1}$ & $0.47_{-0.07}^{+0.08}$ & $190 / 144=1.32$ \\
\hline $0.25-0.50$ & $5.7_{-0.2}^{+0.2}$ & $0.37_{-0.05}^{+0.05}$ & $233 / 226=1.03$ & $5.5_{-0.2}^{+0.2}$ & $0.48_{-0.06}^{+0.06}$ & $260 / 226=1.15$ \\
\hline $0.50-0.83$ & $6.5_{-0.2}^{+0.2}$ & $0.33_{-0.05}^{+0.05}$ & $289 / 233=1.24$ & $6.8_{-0.3}^{+0.3}$ & $0.40_{-0.06}^{+0.06}$ & $243 / 253=0.96$ \\
\hline $0.83-1.67$ & $7.4_{-0.2}^{+0.3}$ & $0.34_{-0.04}^{+0.05}$ & $333 / 343=0.97$ & $7.3_{-0.2}^{+0.2}$ & $0.40_{-0.05}^{+0.05}$ & $429 / 346=1.24$ \\
\hline $1^{\prime} .67-2.50$ & $7.9_{-0.5}^{+0.5}$ & $0.27_{-0.07}^{+0.07}$ & $214 / 195=1.09$ & $7.6_{-0.3}^{+0.5}$ & $0.27_{-0.07}^{+0.07}$ & $237 / 205=1.16$ \\
\hline $2.50-4.17$ & $8.4_{-0.9}^{+0.9}$ & $0.20_{-0.13}^{+0.13}$ & $139 / 108=1.28$ & $8.6_{-0.6}^{+0.6}$ & $0.26_{-0.10}^{+0.11}$ & $202 / 146=1.38$ \\
\hline $4^{\prime} \cdot 17-5 \cdot 83$ & $8.1_{-1.6}^{+2.4}$ & 0.3 (fixed) & $58.9 / 44=1.34$ & $9.1_{-1.9}^{+2.9}$ & 0.3 (fixed) & $50.0 / 39=1.27$ \\
\hline $5: 83-9 ! 17$ & $11.0_{-49}^{+21}$ & 0.3 (fixed) & $40.0 / 26=1.67$ & $6.3_{-18}^{+4.2}$ & 0.3 (fixed) & $71.3 / 40=1.78$ \\
\hline
\end{tabular}

(e.g. $\chi^{2}=0.89-1.21$ with $N_{\mathrm{H}}=3.6 \times 10^{21} \mathrm{~cm}^{-2}$ ), where we found the higher values before, while for EMOS the changes are less significant $\left(\chi^{2}=0.93-1.13\right.$ with $N_{\mathrm{H}}=4.0 \times$ $10^{21} \mathrm{~cm}^{-2}$ ). We should note, however, that some of this improvement of the fit is also due to the fact, that the errors increase for the deprojected spectra.

The fitting results with the lower, free floating $N_{\mathrm{H}}$ values are satisfactory, while the fits at higher $N_{\mathrm{H}}$ are less good. The difference in the best fit $N_{\mathrm{H}}$ value for the two detectors is most probably due to residual problems with the calibration of the detector response matrices. Similar discrepancies were also found in the analysis of other data (e.g. A1795, Tamura et al. 2001). We noticed that the temperature has some difference between the projected and deprojected results in the annular region with 5 ! $8<R<9$ !. 2 in EPN data. This implies that there is still some residual particle background in the projected spectra and it trends to over-determine the projected temperature in the outer regions and makes the determined temperature have relatively high systematic errors.

In all these cases, like the results in the projected spectra, the deprojected spectra also show a temperature drop in the central region (e.g. Fig. 5). In addition, it seems that the temperature also decreases slightly in the outer region $(R>4 . ' 2)$ in the EMOS deprojected spectra (with a significant level $\sim 2.5 \sigma$ ). While for the EPN, this tendency is not so significant due to relatively high statistical and systematic errors. Similarly, the metal abundance is found to decrease from 0.6 in solar units at the center to about 0.2 at a radius of $\sim 300 \mathrm{kpc}$.

To improve the fit for the high literature value of $N_{\mathrm{H}}$, we can alternatively explore the improvement of the fit by adding another cool component with a temperature $T<2 \mathrm{keV}$ to fit the spectra of some of the inner regions. This time we fit the EPN and EMOS spectra simultaneously in order to reduce the errors for each parameter. The results are shown in the Table 4 (also see Fig. 4d). Also the two-component model with the higher $N_{\mathrm{H}}$ value provides formally a satisfactory fit. Thus, without further information, as e.g. a better determination of the absorption column density by some other means, we can not distinguish between the two models. But we note, that the current data do not necessarily require the assumption of a twoor multi-temperature component structure of the ICM.

We also try to fit these spectra with the standard cooling flow model. Because the EMOS may be better than EPN in the determination of the galactic absorption $N_{\mathrm{H}}$ (see Molendi \& Pizzolato 2001), we only use the EMOS spectra to investigate the cooling flow. We adopt a cooling model (Mushotzky \& Szymkowiak 1988) of the form wabs(mekal+zwabs(mkcflow)) in XSPEC. The $N_{\mathrm{H}}$ value is fixed to the galactic value $4.24 \times$ $10^{21} \mathrm{~cm}^{-2}$ and we set the low temperature in mkcflow to $0.01 \mathrm{keV}$. Since the best fit value of the intrinsic absorption in zwabs always turns to be 0 when we set it free in the fit, we fix it to 0 . Therefore, there are in total 4 free parameters in this model, i.e. the isothermal temperature $T$, the abundance, the normalization value of the mekal model and the cooling flow rate. The inner 4 annular spectra were fitted with the above model one by one and then we sum the cooling flow rate of all regions together. The total cooling flow is found to be $364_{-94}^{+97} M_{\odot} \mathrm{yr}^{-1}$ (Table 5), which is much smaller than the previously obtained value, e.g. $1455_{-510}^{+356} M_{\odot} \mathrm{yr}^{-1}$ (Allen 2000). We'd like to emphasis that the cooling flow rate is sensitive to the value of $N_{\mathrm{H}}$. If $N_{\mathrm{H}}=4.0 \times 10^{21} \mathrm{~cm}^{-2}$ is assumed, the total mass-flow rate becomes $176_{-75}^{+88} M_{\odot} \mathrm{yr}^{-1}$ and the results are also consistent with an insignificant cooling flow rate. This result is analogous to the analysis with the two-temperature model above.

We also fitted the deprojected EMOS spectra from the region with $R<1$ '.67 with the standard cooling flow model wabs(mekal+zwabs(mkcflow)) (Fig. 6). Here the value of $N_{\mathrm{H}}$ is fixed to $4.24 \times 10^{21} \mathrm{~cm}^{-2}$ and the intrinsic absorption in zwabs is set to be free. The resultant cooling flow rate is about $408_{-44}^{+113} M_{\odot} \mathrm{yr}^{-1}$. When we further fixed the cooling flow rate to $1000 M_{\odot} \mathrm{yr}^{-1}$, the value of reduced $\chi^{2}$ changed from 1.25 (d.o.f. $=399$ ) to 1.34 (d.o.f. $=400$ ). The null hypothesis probability from XSPEC, which means the probability of getting a value of $\chi^{2}$ larger than observed if the model is correct, is $4.8 \times 10^{-6}$. Moreover, the simple F-test (Bevington 1969) shows that the change of the reduced $\chi^{2}$ is significant at $\gg 99.9 \%$ confidence. These results indicate that the large cooling flow rate $\left(\sim 1000 \mathrm{M}_{\odot} \mathrm{yr}^{-1}\right)$ can be excluded. 

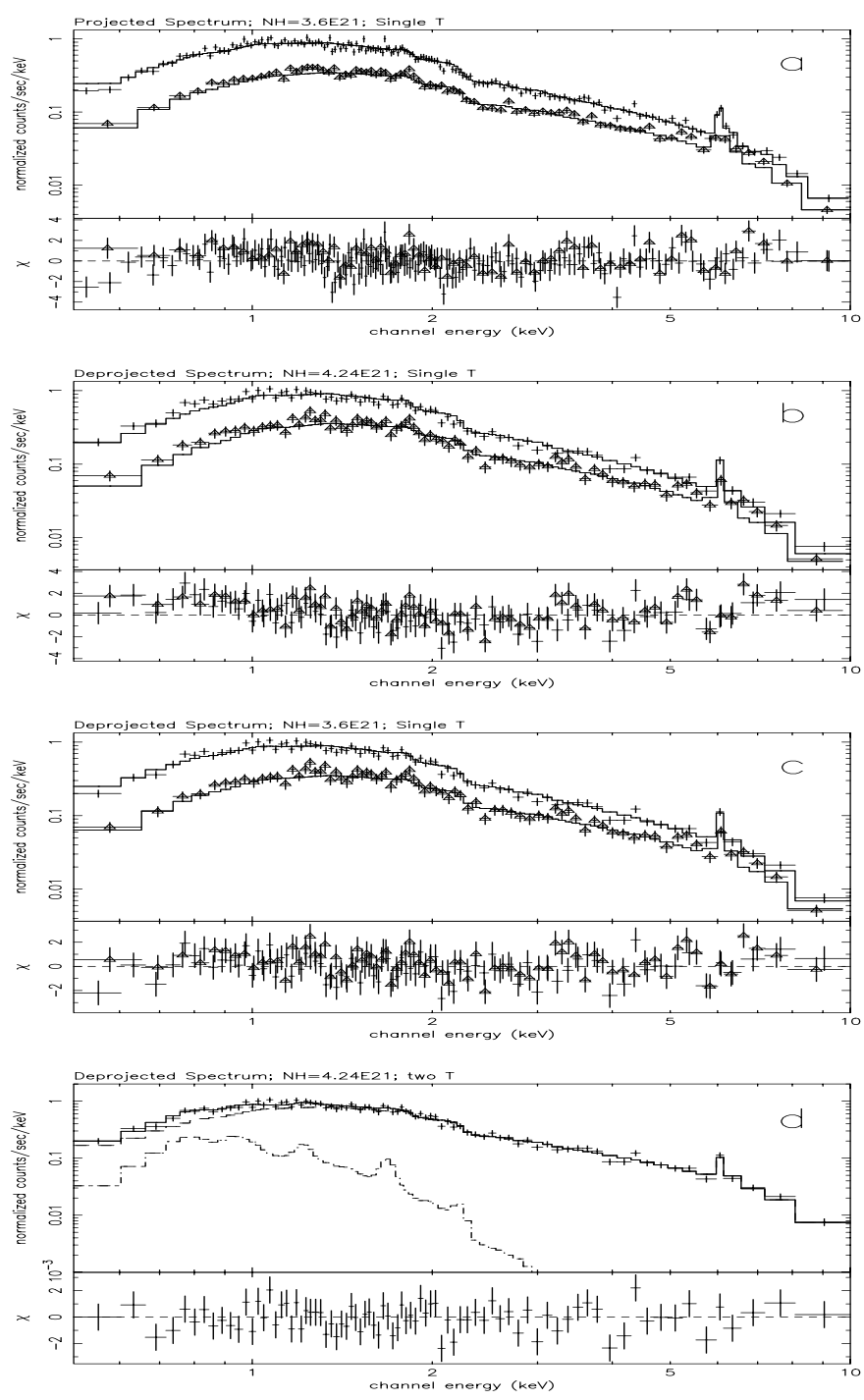

Fig. 4. Spectra from EPN for a representative annular region with a radius $0.50<R<0$ '.83. Crosses represent the observed spectra of single events (PATTERN $=0$ ) of EPN and triangles represent double events $(1 \leq$ PATTERN $\leq 4)$. Solid lines represent the best-fit models. a) Projected spectra fitted with a single temperature Mekal model with $N_{\mathrm{H}}=3.6 \times 10^{21} \mathrm{~cm}^{-2}$. b) Deprojected spectra fitted with a single temperature Mekal model with $N_{\mathrm{H}}=4.24 \times 10^{21} \mathrm{~cm}^{-2}$. c) Same as b) but with $N_{\mathrm{H}}=3.6 \times 10^{21} \mathrm{~cm}^{-2}$. d) Same as b) but with a two-component Mekal model and only single events are shown for clarity. The cool component is shown in a dashed-dot line, hot component in a dashed line and total model in a solid line. Note the fitting results in c) and d) give better $\chi^{2}$ values (see Tables $2-4$ ).

\section{Morphology of the cluster center}

In the central region of the cluster, the X-ray emission peak is found to be close to the radio peak in the image from the radio observations at 6 and $20 \mathrm{~cm}$ obtained with the VLA A (Baum \& O'Dea 1991; see Fig. 7). Since the central cluster galaxy contains an active galactic nucleus, we first check if we can detect any X-ray emission from this AGN, which would have also contaminated the X-ray spectrum of the innermost region. We here present an X-ray image from Chandra with observation ID 508, in which we found no bright X-ray point like source in the center of the cluster. Furthermore, we can see
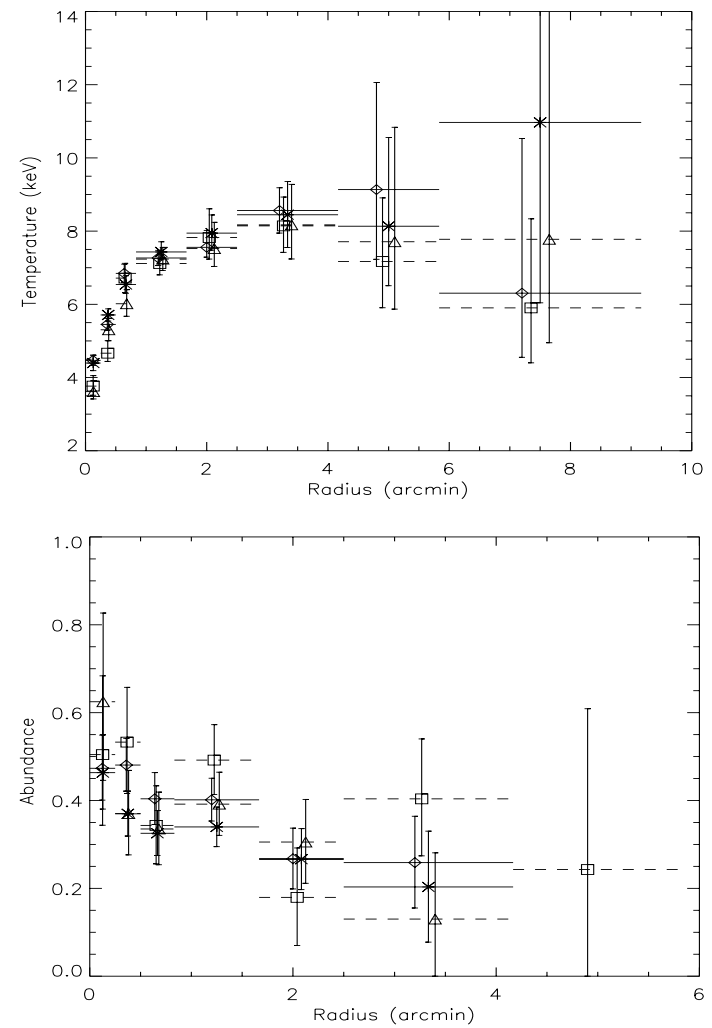

Fig. 5. Radial temperature profile (top panel) and abundance profile (bottom panel). Solid lines represent projected results and dashed lines are deprojected results. Crosses and triangles represent data from EPN. Diamonds and rectangles are from EMOS. Note that here the $N_{\mathrm{H}}$ value is fixed to $3.6 \times 10^{21} \mathrm{~cm}^{-2}$ and $4.0 \times 10^{21} \mathrm{~cm}^{-2}$ for EPN and EMOS, respectively.

that in the Chandra image the X-ray and radio peak coincide even better (Fig. 7). There is also an enhanced diffuse X-ray emission region to the west, which is located just outside the 6-cm radio emission region (Fig. 7, medium panel). This structure may be associated with a buoyant plasma bubble.

For the XMM-Newton data, we selected a radio bright polygon region (see Fig. 7, top panel) and compared the spectrum inside with that from the ambient region taken from a circular region with a radius $R=0.25$ excluding the above polygon region. In this analysis, we fit each spectrum with a single temperature Mekal model (see Table 6). The results show that the temperature in the region of radio source is lower than that in the ambient region with a statistical significant level $\sim 2.2 \sigma$. Since the area of the polygon region is not very large compared with the scale $\left(F W H M 6^{\prime \prime}\right)$ of the Point-Spread Function (PSF) of XMM, we should consider the PSF effect here. However, the $F W H M$ of the PSF of hard X-ray photons is smaller than that of soft X-ray photons (Ehle et al. 2001). Thus the fitted temperature in the brighter region trends to be higher than that in its nearby regions, because the brighter region loses some net soft photons. This effect is also confirmed by numerical simulations. Thus, in our case, the discrepancy of the temperature can not arise from the energy dependence of the PSF. Furthermore, this may be an indication of an interaction effect of the radio lobes with the ICM, similar to the one found in M 87 (Böhringer et al. 1995; Belsole et al. 2001) where the 
Table 3. Fit of deprojected spectra with a single temperature model and $N_{\mathrm{H}}=4.24 \times 10^{21} \mathrm{~cm}^{-2}$ for both EPN and EMOS.

\begin{tabular}{|c|c|c|c|c|c|c|}
\hline Radius & $T(\mathrm{keV})$ & Abundance & $\chi^{2}$ & $T(\mathrm{keV})$ & Abundance & $\chi^{2}$ \\
\hline Instrument & & EPN & & & EMOS & \\
\hline $0^{\prime}-0.25$ & $3.3_{-0.2}^{+0.2}$ & $0.64_{-0.17}^{+0.20}$ & $106 / 77=1.38$ & $3.6_{-0.2}^{+0.3}$ & $0.50_{-0.16}^{+0.18}$ & $81.5 / 81=1.01$ \\
\hline $0.25-0.50$ & $4.6_{-0.2}^{+0.3}$ & $0.38_{-0.09}^{+0.09}$ & $196 / 183=1.07$ & $4.5_{-0.2}^{+0.2}$ & $0.54_{-0.12}^{+0.12}$ & $160 / 163=0.98$ \\
\hline $0.50-0,83$ & $5.2_{-0.3}^{+0.3}$ & $0.34_{-0.08}^{+0.08}$ & $231 / 158=1.46$ & $6.3_{-0.4}^{+0.4}$ & $0.34_{-0.08}^{+0.09}$ & $238 / 246=0.97$ \\
\hline $0.83-1.67$ & $6.3_{-0.3}^{+0.3}$ & $0.36_{-0.06}^{+0.06}$ & $223 / 173=1.29$ & $6.7_{-0.3}^{+0.3}$ & $0.48_{-0.07}^{+0.08}$ & $212 / 177=1.20$ \\
\hline $1^{\prime} .67-2.50$ & $6.5_{-0.5}^{+0.5}$ & $0.29_{-0.08}^{+0.008}$ & $238 / 225=1.06$ & $7.3_{-0.5}^{+0.7}$ & $0.18_{-0.10}^{+0.11}$ & $228 / 231=0.99$ \\
\hline $2.50-4.17$ & $6.7_{-0.7}^{+0.7}$ & $0.16_{-0.13}^{+0.13}$ & $161 / 169=0.95$ & $7.5_{-0.5}^{+0.8}$ & $0.39_{-0.12}^{+0.12}$ & $235 / 226=1.04$ \\
\hline $4.17-5.83$ & $6.3_{-1.4}^{+2.0}$ & 0.3 (fixed) & $62.8 / 59=1.06$ & $6.6_{-1.0}^{+1.5}$ & $0.25_{-0.25}^{+0.35}$ & $122 / 115=1.06$ \\
\hline $5.83-9.17$ & $6.3_{-2.1}^{+4.6}$ & 0.3 (fixed) & $67.9 / 60=1.13$ & $5.5_{-1.4}^{+2.1}$ & $0.64_{-0.17}^{+0.20}$ & $78.0 / 73=1.05$ \\
\hline
\end{tabular}

Table 4. Simultaneous fit of EPN and EMOS spectra with a two-component model and with $N_{\mathrm{H}}=4.24 \times 10^{21} \mathrm{~cm}^{-2}$.

\begin{tabular}{cccccccc}
\hline \hline Radius & $T_{\text {hot }}(\mathrm{keV})$ & $T_{\text {cool }}(\mathrm{keV})$ & Abundance & Nom $_{\text {hot }}$ & Nom $_{\text {cool }}$ & $f_{\text {vol }}{ }^{a}$ & $\chi^{2}$ \\
\hline $0^{\prime}-0.25$ & $5.3_{-1.5}^{+3.6}$ & $1.4_{-0.6}^{+0.4}$ & $0.32_{-0.14}^{+0.32}$ & $2.4_{-0.8}^{+0.7} \times 10^{-3}$ & $1.2_{-1.0}^{+1.2} \times 10^{-3}$ & $3.3 \times 10^{-2}$ & $155 / 158=0.98$ \\
$0^{\prime} 25-0^{\prime} 50$ & $4.7_{-0.2}^{+0.3}$ & $0.27_{-0.06}^{+0.09}$ & $0.44_{-0.07}^{+0.08}$ & $7.7_{-0.2}^{+0.3} \times 10^{-3}$ & $5.4_{-3.6}^{+3.6} \times 10^{-4}$ & $2.3 \times 10^{-4}$ & $342 / 346=0.99$ \\
$0^{\prime} 50-0^{\prime} 83$ & $6.3_{-0.3}^{+0.4}$ & $0.62_{-0.14}^{+0.17}$ & $0.33_{-0.06}^{+0.06}$ & $9.6_{-0.2}^{+0.3} \times 10^{-3}$ & $6.5_{-1.8}^{+1.7} \times 10^{-4}$ & $6.6 \times 10^{-4}$ & $431 / 404=1.07$ \\
$0^{\prime} 83-1^{\prime} 67$ & $7.0_{-0.3}^{+0.2}$ & $0.60_{-0.29}^{+0.09}$ & $0.43_{-0.05}^{+0.05}$ & $1.40_{-0.02}^{+0.03} \times 10^{-2}$ & $5.7_{-1.2}^{+1.6} \times 10^{-4}$ & $3.0 \times 10^{-4}$ & $385 / 350=1.10$ \\
\hline
\end{tabular}

${ }^{a} f_{\text {vol }}$ is the volume filling fraction of the cooler component and $f_{\text {vol }}=\left[1+\left(\operatorname{Nom}_{\text {hot }} / \operatorname{Nom}_{\text {cool }}\right)\left(T_{\text {hot }} / T_{\text {cool }}\right)^{2}\right]^{-1}$.

temperature in the radio lobe regions is found to be lower than in the ambient plasma.

\section{Mass determination}

\subsection{Projected mass within critical radius}

A strongly lensed arc with a radius $r_{\mathrm{c}}=45.9 \mathrm{kpc}$ has been found in the cluster PKS 0745-191 which can be used to constrain the cluster mass profile. In fact, from the deprojected spectra, one can determine the mass distribution in the cluster. However, the mass value is sensitive to the value of the derivative $\mathrm{d}(n T) / \mathrm{d} r$ ( $n$ is the electron number density or equivalently gas density). We deproject the radial surface brightness distribution assuming spherical symmetry and determine the electron number density distribution profile (Fig. 8). Next we fit the electron number density with a $\beta$ model (Cavaliere \& Fusco-Femiano 1976) in which the electron number density is defined as:

$n(r)=n_{0}\left(1+\left(r / r_{\mathrm{c}}\right)^{2}\right)^{-3 \beta / 2}$,

where $n_{0}$ is central number density and $r_{\mathrm{c}}$ is the core radius. The resultant fit parameters are $n_{0}=0.055 \pm 0.001 \mathrm{~cm}^{-3}$, $r_{\mathrm{c}}=62 \pm 1 \mathrm{kpc}$ and $\beta=0.572 \pm 0.004$. We fit the deprojected radial temperature with an exponential function. At last, the mass distribution (Fig. 9, top panel) can be derived from these two functions, assuming the gas is in hydrostatic equilibrium with the cluster potential. Using this method, we also obtained the projected mass within the critical radius $M_{\mathrm{c}}=$ $1.10 \pm 0.04 \times 10^{13} M_{\odot}$ (Fig. 9, bottom panel), which is roughly a factor $\sim 2$ lower than that from optical lensing (Allen et al. 1996). We should note that this discrepancy can not be resolved by the two-component model described in Sect. 3.2 (see
Table 4). In fact, when we adopt the two-component model, the obtained total mass decreases to about $1.0 \times 10^{13} M_{\odot}$. We explore a possible reason for this discrepancy in Sect. 6 .

We also fitted the electron number density profile with a double $\beta$ model as below:

$n(r)=n_{01}\left(1+\left(r / r_{\mathrm{c} 1}\right)^{2}\right)^{-3 \beta_{1} / 2}+n_{02}\left(1+\left(r / r_{\mathrm{c} 2}\right)^{2}\right)^{-3 \beta_{2} / 2}$,

from which we obtained the best fit values as follows: $n_{01}=$ $0.054 \pm 0.004 \mathrm{~cm}^{-3}, n_{02}=0.012 \pm 0.002 \mathrm{~cm}^{-3}, \beta_{1}=0.74 \pm$ $0.11, \beta_{2}=0.63 \pm 0.01, r_{\mathrm{c} 1}=47 \pm 2 \mathrm{kpc}, r_{\mathrm{c} 2}=161 \pm 14 \mathrm{kpc}$. The resultant value of $M_{\mathrm{c}}$ increases only a little to $1.18 \pm 0.04 \times$ $10^{13} M_{\odot}$ when we use the double $\beta$ model.

\subsection{Gas mass fraction}

Similar to the work on the cluster Abell 1835 (Majerowicz et al. 2002) and Abell 1413 (Pratt \& Arnaud 2002), we also calculated the gas mass fraction of PKS 0745 and show the profile in Fig. 10. Here we use the double $\beta$ model to fit the electron number density. The virial radius of the cluster, $r_{\mathrm{vir}}$, can be calculated from (Evrard et al. 1996; Majerowicz et al. 2002):

$r_{\mathrm{vir}}=3690 \sqrt{T / 10}(1+z)^{-3 / 2} \mathrm{kpc}$,

where $T$ is the mean temperature of the cluster in $\mathrm{keV}$. We adopted a fitted temperature of $7.7 \mathrm{keV}$ and obtained $r_{\text {vir }}=$ $2.8 \mathrm{Mpc}$. At last, we obtain a mean gas mass fraction $f_{\text {gas }}=$ $0.22 \pm 0.02$ within $11.6\left(0.63 r_{\text {vir }}\right)$. This result is comparable to that from other cluster, e.g. $f_{\text {gas }}=0.207 \pm 0.037$ (Abell 1835; Majerowicz et al. 2002) and $f_{\text {gas }} \sim 0.2$ (Abell 1413; Pratt \& Arnaud 2002). The good agreement in the gas mass fraction with other observations rules out the possibility that the mass 
Table 5. Cooling flow rate of each region from EMOS data with $N_{\mathrm{H}}=4.24 \times 10^{21} \mathrm{~cm}^{-2}$.

\begin{tabular}{|c|c|c|c|c|}
\hline Radius & Radius (kpc) & $T(\mathrm{keV})$ & Cooling flow rate $\left(M_{\odot} \mathrm{yr}^{-1}\right)$ & $\chi^{2}$ \\
\hline $0^{\prime}-0.25$ & $0-37.8$ & $4.4_{-0.5}^{+1.0}$ & $85_{-38}^{+45}$ & $67.8 / 80=0.85$ \\
\hline $0.25-0.50$ & $37.8-75.5$ & $4.8_{-0.4}^{+0.6}$ & $47_{-47}^{+54}$ & $159 / 162=0.98$ \\
\hline $0.50-0.83$ & $75.5-125.8$ & $7.7_{-0.9}^{+1.4}$ & $119_{-37}^{+56}$ & $228 / 245=0.93$ \\
\hline $0.83-1.67$ & $125.8-251.7$ & $7.5_{-0.5}^{+1.0}$ & $113_{-61}^{+37}$ & $201 / 176=1.14$ \\
\hline $0^{\prime}-1.67$ & $0-251.7$ & - & $364_{-94}^{+97}$ & - \\
\hline
\end{tabular}

Table 6. Comparison of spectra in central region.

\begin{tabular}{cccccc}
\hline \hline Region & Instrument & $N_{\mathrm{H}}\left(10^{21} \mathrm{~cm}^{-2}\right)$ & $T(\mathrm{keV})$ & Abundance & $\chi^{2} /$ d.o.f. \\
\hline Radio & EPN & 3.6 (fixed) & $3.3_{-0.3}^{+0.3}$ & $0.66_{-0.28}^{+0.36}$ & $28.8 / 23=1.25$ \\
Ambient & EPN & 3.6 (fixed) & $3.6_{-0.4}^{+0.5}$ & $0.73_{-0.35}^{+0.44}$ & $25.5 / 25=1.02$ \\
Radio & EMOS & 4.0 (fixed) & $3.5_{-0.2}^{+0.2}$ & $0.73_{-0.26}^{+0.31}$ & $44.1 / 39=1.13$ \\
Ambient & EMOS & 4.0 (fixed) & $4.1_{-0.4}^{+0.5}$ & $0.38_{-0.27}^{+0.32}$ & $45.1 / 42=1.07$ \\
\hline
\end{tabular}
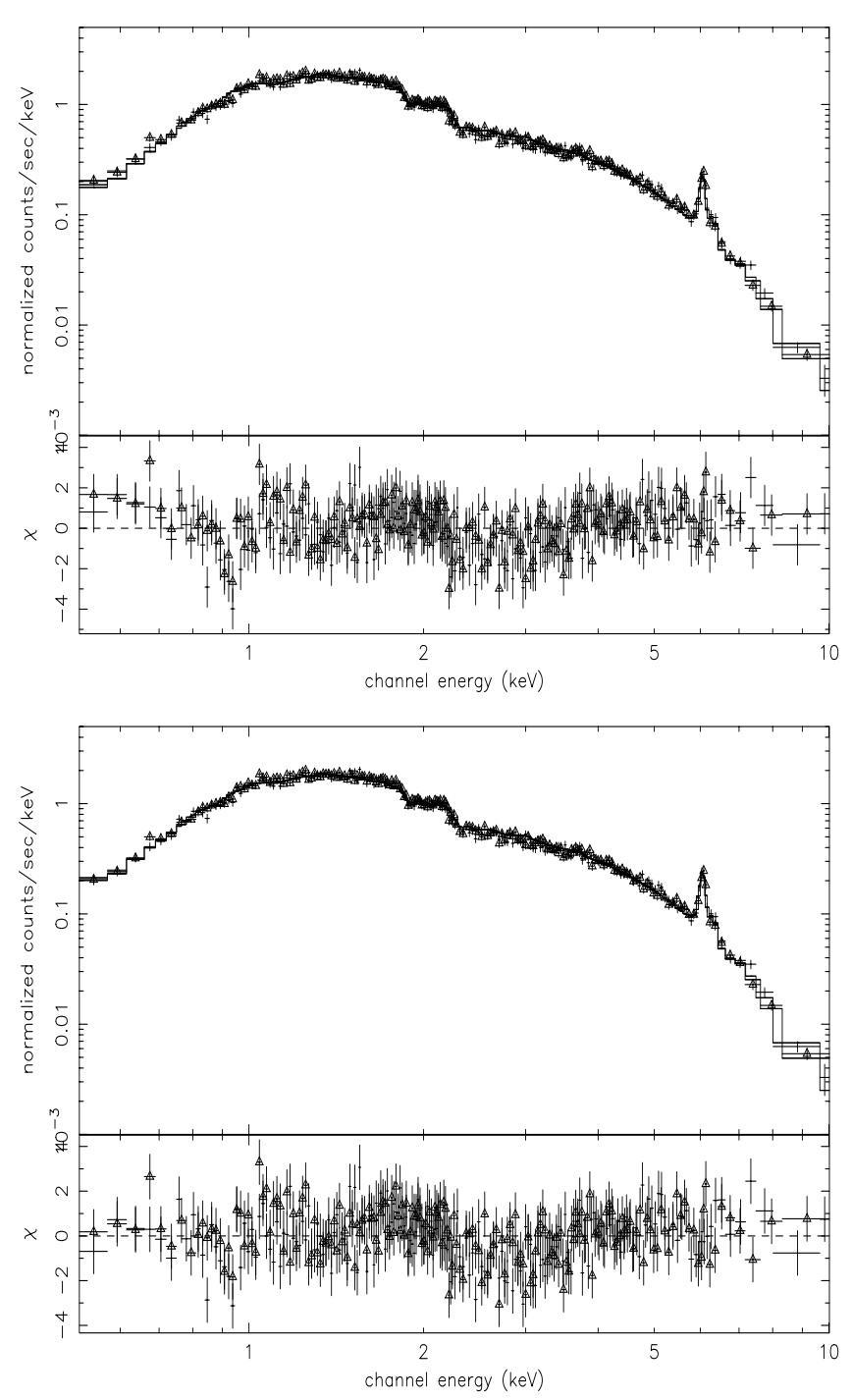

Fig. 6. Top panel: Deprojected EMOS spectra fitted with a standard cooling flow model with the parameter of cooling flow rate fixed to $1000 M_{\odot} \mathrm{yr}^{-1}$. Crosses represent the data from EMOS1 and triangles are from EMOS2. Note the much larger residuals in the top panel at low energy. Bottom panel: Same as top panel but the cooling flow rate is a free parameter. determination from the X-ray data is incorrect on a global scale. The gass mass profile is derived in a very direct way from the surface brightness distribution and expected to be quite precise. Therefore a deficiency in the mass determination giving rise to the above described discrepancy should be restricted to the very central region of the cluster (may be the region where we also observed a strange rise in the gas mass fraction in Fig. 10).

\section{Discussions and summary}

In the background subtraction, we use an annular region with a radius 11 . $6<R<15$ ! 0 to determine the additional background. However, in this region, there should be still some emission from the cluster. When we subtract it from the inner spectra, it may cause some systematic errors. We can estimate the emission of cluster in this region by the off-set data. Similar to the above background subtraction for the central observational data, in step 1 , we use "closed" observational data (observational ID 0122320701) as the background data and use an annular region with a radius 20 . $^{\prime} 0<R<25$. 0 to the cluster center to determine the sky background in step 3 . Then we can get the surface brightness profile of the off-set data. Furthermore, if we assume the cluster surface brightness in the same sky region with $8.5<R<15$ '. 0 is the same between the two observations, we can obtain the combined surface brightness of the cluster (Fig. 11). The surface brightness of the cluster in a region with $11.6<R<15$ ! 0 is found to be very small compared with the background in the same region (Table 7) and, for example, it is only a few percent of the surface brightness in the region with $R \sim 5$ '.0. So it is reasonable for us to use this region to determine the additional background.

The existence of strong and diffused radio emission implies that there is a large amount of relativistic plasma in the center of the cluster. As discussed in Sect. 4, there is an enhanced diffuse X-ray emission region near the center of the cluster. First we investigated if inverse-Compton emission from the relativistic electrons and photons of the microwave background (Harris \& Grindlay 1979) can contribute significantly to the observed $\mathrm{X}$-ray emission. The inverse-Compton emission flux in X-ray 
Table 7. The background flux compared with the inferred cluster emission flux of EPN in the $11^{\prime} \cdot 6-15^{\prime} .0$ region.

\begin{tabular}{ccc}
\hline \hline Total flux $(0.3-10.0 \mathrm{keV})$ after step 2 & cluster flux $(0.5-5.0 \mathrm{keV})$ & cluster flux $(0.3-10.0 \mathrm{keV})$ \\
\hline$\left(\right.$ counts $\left./ \mathrm{s} / \mathrm{arcmin}^{2}\right)$ & $\left(\right.$ counts $\left./ \mathrm{s} / \mathrm{arcmin}^{2}\right)$ & $\left(\right.$ counts $\left./ \mathrm{s} / \mathrm{arcmin}^{2}\right)$ \\
$7.3 \pm 0.3 \times 10^{-3}$ & $4.5 \pm 0.3 \times 10^{-4}$ & $6.9 \pm 0.5 \times 10^{-4}$ \\
\hline
\end{tabular}
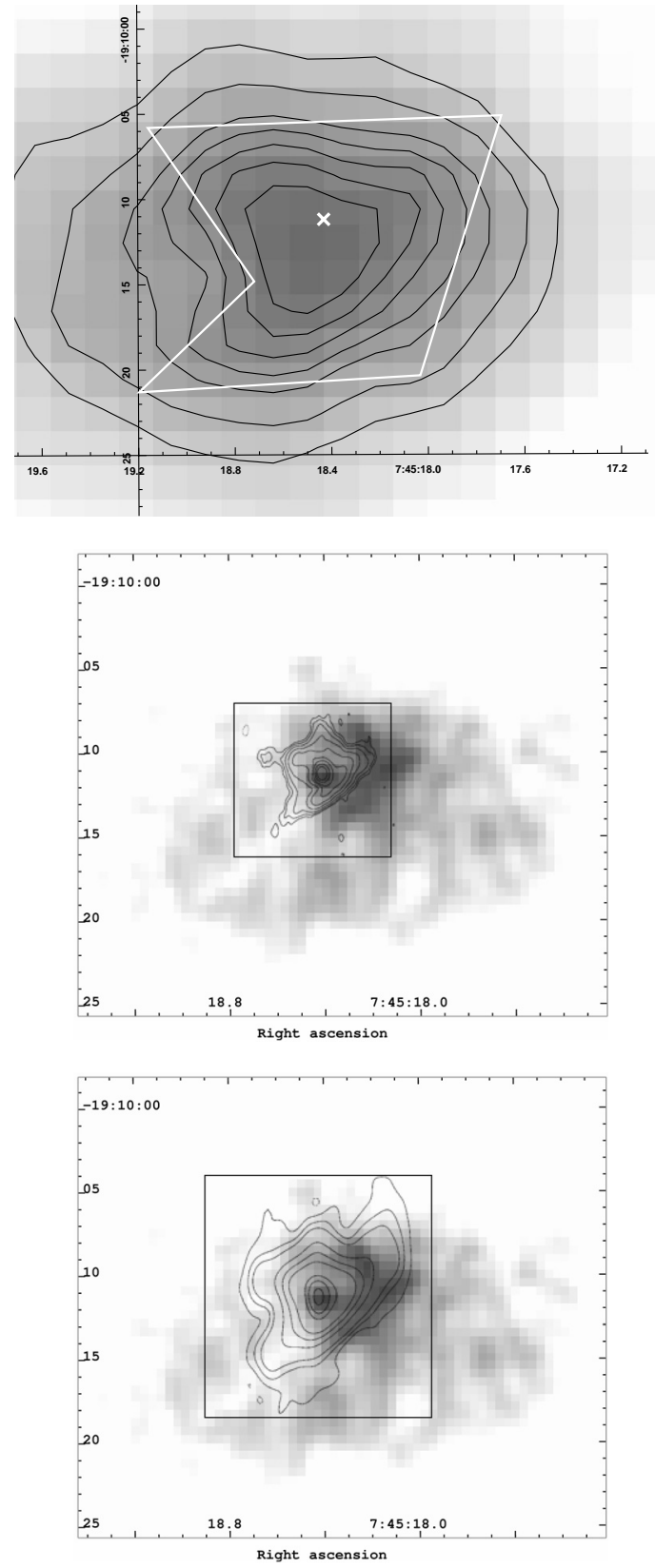

Fig. 7. Top panel: Central region EPN image in B1950 coordinates with $2^{\prime \prime} \times 2^{\prime \prime}$ per pixel. The image has been smoothed with a Gaussian of $\sigma=2^{\prime \prime}$ and contours are at $(80,90,100,105,110$, $115,120)$ counts/pixel. The center spectrum is got from the polygon region and the cross shows the emission peak of the radio image map. Medium panel: Chandra image with pixel size 0.'5 $\times 0.0^{\prime} 5$ and with 9-23 counts/pixel in the central box region. The image has been smoothed with a Gaussian of $\sigma=00^{\prime} 5$ overlaid with 6-cm VLA A array contours (Baum \& O'Dea 1991). The radio beam size is $0.59 \times$ $0.37 \mathrm{arcsec}^{2}$. Bottom panel: Same as medium panel but with $20-\mathrm{cm}$ VLA A array contours. The radio beam size is $1.76 \times 1.01 \operatorname{arcsec}^{2}$.

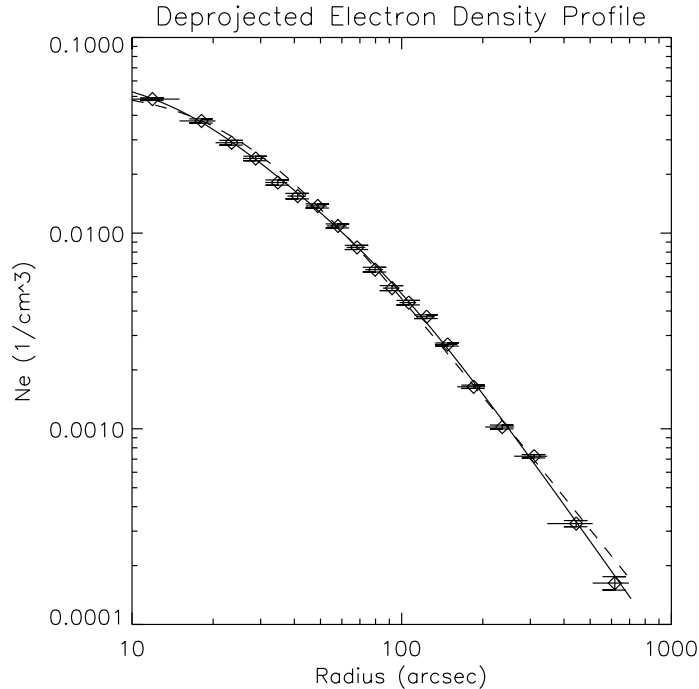

Fig. 8. The deprojected electron number density as a function of radius from the cluster center. The fit curve using a $\beta$ model is shown as a dashed line and a double $\beta$ model as a solid line.
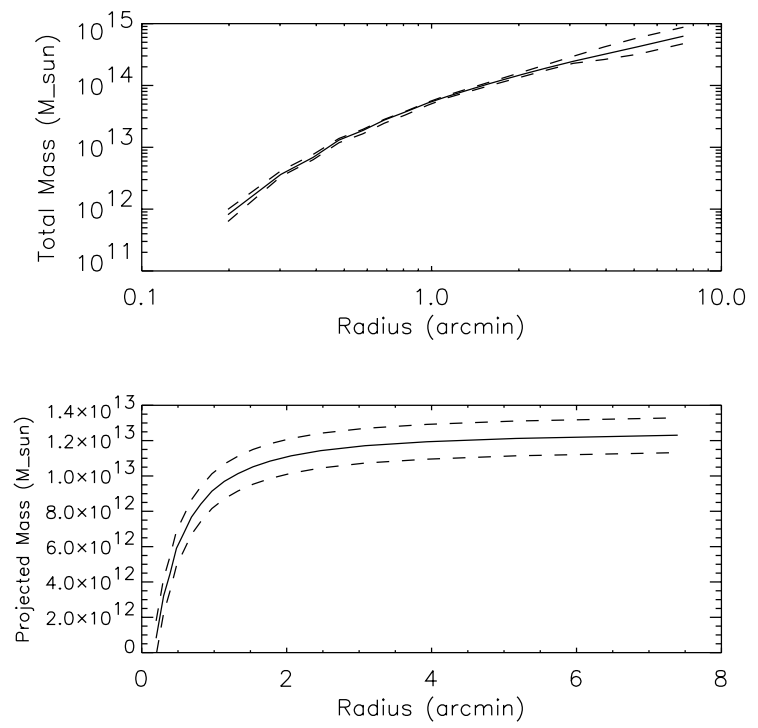

Fig. 9. Top panel: Gravitational mass distribution. Bottom panel: Total projected mass within the critical radius as a function of the cluster radius. The critical radius of the gravitational arc is at $45.9 \mathrm{kpc}(0.3)$. Dashed lines are at $1 \sigma$ significance.

can be calculated with some parameters of the radio source determined by Baum \& O’Dea (1991) as below:

$f_{x}=C(\alpha) S_{\mathrm{r}}(1+z)^{5 / 2} E_{\mathrm{c}}^{3 / 2} F(\alpha, E) /\left(v_{\mathrm{r}} B\right)^{1 / 2}$,

where $f_{x}$ is the predicted inverse-Compton X-ray flux, $E_{\mathrm{c}} \sim 5.0 \mathrm{keV}$ is the center of the observed X-ray energy 


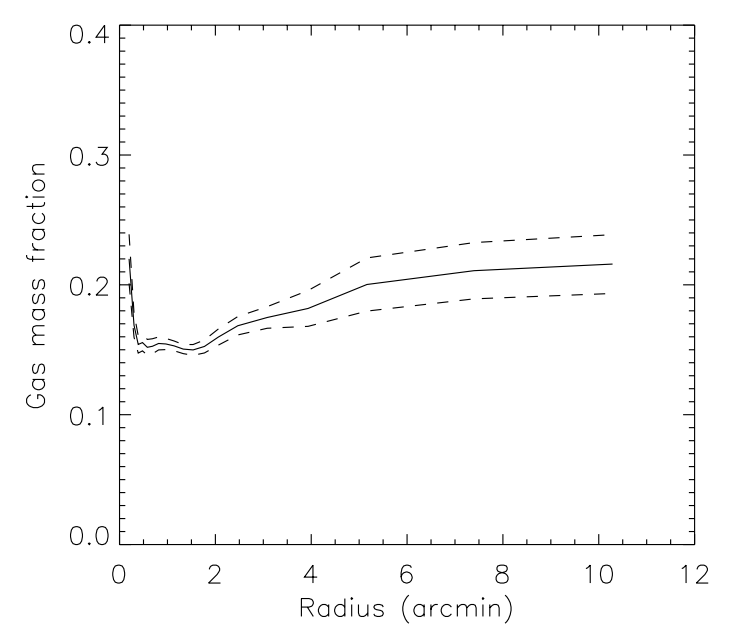

Fig. 10. Gas mass fraction profile calculated with a double $\beta$ model. Dashed lines are at $1 \sigma$ significance.

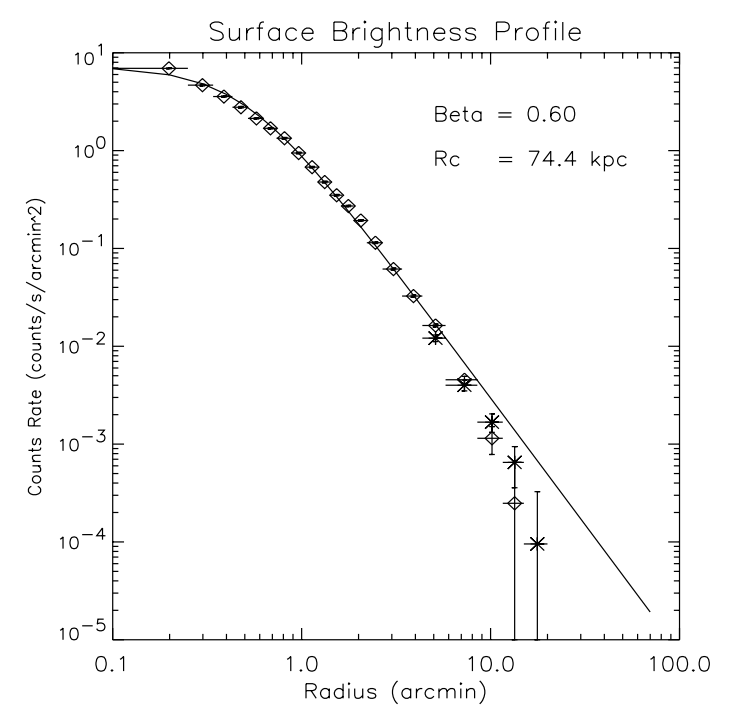

Fig. 11. A combined surface brightness profile of the cluster. The fit curve using a $\beta$ model is shown as a solid line. Diamonds represent data from central observational data and crosses are from off-set observational data.

band, $\alpha \sim 1.4$ is the power law exponent of the radio spectrum, $S_{\mathrm{r}} \sim 2.0 \times 10^{-23} \mathrm{erg} \mathrm{cm}^{-2} \mathrm{~s}^{-1} \mathrm{~Hz}^{-1}$ is the observed flux density of the radio source at frequency $\nu_{\mathrm{r}}=1490 \mathrm{MHz}$, $C(\alpha) \sim 6.1 \times 10^{7}$ for the range $0.5<\alpha<2.0, B \sim 20 \mu \mathrm{G}$ is the magnetic field corresponding to minimum pressure in a diffuse plume of the radio source, $F(\alpha, E) \sim 1.3$ for $E=5.0 \mathrm{keV}$ and $\alpha=1.4$. Thus we find $f_{x} \sim 1.3 \times 10^{-16} \mathrm{ergs} \mathrm{cm}^{-2} \mathrm{~s}^{-1}$. While from the central spectrum, the total X-ray flux is about $1.5 \times$ $10^{-12} \mathrm{ergs} \mathrm{cm}^{-2} \mathrm{~s}^{-1}$. The result shows that the inverse-Compton flux is very small, about 4 orders of magnitude lower than the total observed X-ray flux.

Now we investigate another possibility. The relativistic plasma may entrain some thermal plasma and form some buoyant bubbles (Sarazin et al. 1995; Churazov et al. 2001). When the bubbles lift, the entrained thermal gas will expand adiabatically and the emission may be enhanced (Churazov et al. 2001;
Böhringer et al. 2002) compared with ambient regions. This can explain the enhanced X-ray emission in the inner regions.

The pressure of the X-ray gas in the central region can be calculated from:

$P_{\text {gas }}=2 n_{\mathrm{e}} k T$,

where $n_{\mathrm{e}}$ is the electron number density of the X-ray gas. With $n_{\mathrm{e}}=0.04 \mathrm{~cm}^{-3}$ and $T=4 \mathrm{keV}$, we got $P_{\text {gas }}=$ $5 \times 10^{-10}$ dyne $\mathrm{cm}^{-2}$. This value is one order of magnitude larger than the minimum pressure in the radio plasma $(5 \times$ $10^{-11}$ dyne $\mathrm{cm}^{-2}$, see Baum \& O'Dea 1991). This result implies that there is lots of energy in relativistic protons in the central region (Baum \& O’Dea 1991). Since we do not know the relative energy density of electrons and protons and since the energy density in protons might be much higher than that of the electrons like in our galaxy, the pressure of protons is very likely to be important in the central region of the cluster.

Moreover, if there is lots of energy in relativistic protons in the central region of the cluster as suggested by Baum \& O'Dea (1991), they can provide some additional pressure and occupy some volume. In fact, in Fig. 5 medium panel, there is some evidence that the radio plasma occupies some volume in the central region of the cluster, where the X-ray emission is lower than that in the center and the enhanced emission region. This may also explain the discrepancy of the obtained gravitational mass between X-ray and optical lens. In fact, when we assume that the relativistic protons occupy a major volume in the central region with a radius, e.g. $R<1^{\prime}$ or $R<0$ ' 3 , the resultant mass can be consistent with that from the optical lens method.

This mass discrepancy between the X-ray and lensing data was inferred previously with a similar magnitude from the analysis of ASCA data (Allen et al. 1996) for single temperature models. The X-ray determined mass in the central region was, however, increased by adopting a multi-temperature cooling flow model and claimed to be consistent with the lensing mass. The present results can now definitely rule out this possibility. We further found no increase in the inferred mass when we included the smaller implied cooling flow in our mass analysis. We therefore conclude that the implied mass discrepancy has another reason but an inappropriate cooling flow treatment and we may have found the true cause as explained above.

We have presented the analysis of XMM-Newton observations of the cluster of galaxies PKS 0745-191. The main results of this work may be summarized as follows:

1. We trace the temperature drop towards the center with direct data for the first time and show that it is a monotonic, smooth transition. The earlier results did not have the resolution to provide these results in a unique way. We also show that the results are close to be single phased in temperature outside the inner 0.25 , and that even though we cannot rule out a second component, this component has a low importance characterized by an emission measure of less than 10 percent (see Table 4).

2. The spectral analysis is consistent with a cooling flow mass deposition rate of $364_{-94}^{+97} M_{\odot} \mathrm{yr}^{-1}$ and we can exclude a strong cooling flow $\left(\sim 1000 \mathrm{M}_{\odot} \mathrm{yr}^{-1}\right)$ with high confidence. We also find no evidence for excess internal absorption usually associated with the previous cooling flow results (Allen et al. 2001). 
3. We find that the asymmetric X-ray emission in the core region is associated with the radio lobes. We explore possible implications on the interactions between the radio lobes and the intracluster plasma.

4. We find a total mass of about $1.18 \pm 0.04 \times 10^{13} M_{\odot}$ within the critical radius of the observed gravitational arc, which is a factor $\sim 2$ lower, however, than that from optical lensing.

5 . We calculate the gas mass fraction profile up to $0.63 r_{\text {vir }}$ and obtain a mean gas mass fraction $f_{\text {gas }}=0.22 \pm 0.02$.

Acknowledgements. The paper is based on observations obtained with XMM-Newton, an ESA science mission with instruments and contributions directly funded by ESA Member States and the USA (NASA). The XMM-Newton project is supported by the Bundesministerium für Bildung und Forschung, Deutsches Zentrum für Luft und Raumfahrt (BMBF/DLR), the Max-Planck Society and the Haidenhain-Stiftung. Y. Chen was supported by the exchange program between the Max-Planck Society and the Chinese Academy of Sciences. He thanks MPE for its very gracious hospitality. This research is partially supported by the Special Funds for Major State Basic Research Projects and the National Natural Science Foundation of China.

\section{References}

Allen, S. W., Fabian, A. C., \& Kneib, J. P. 1996, MNRAS, 279, 615

Allen, S. W. 1998, MNRAS, 296, 392

Allen, S. W. 2000, MNRAS, 315, 269

Allen, S. W., Ettori, S., \& Fabian, A. C. 2001, MNRAS, 324, 877

Arnaud, K. A. 1996, ADASS, 101, 5

Baum, S. A., \& O'Dea, C. P. 1991, MNRAS, 250, 737

Belsole, E., Sauvageot, J. L., Böhringer, H., et al. 2001, A\&A, 365, L188

Bevington, P. R. 1969, Data reduction and Error analysis for the Physical Sciences (New York: McGraw-Hill)

Böhringer, H., Voges, W., Fabian, A. C., Edge, A. C. \& Neumann, D. M. 1993, MNRAS, 264, 25

Böhringer, H., Nulsen, P. E. J., Braun, R., \& Fabian, A. C. 1995, MNRAS, 274, L67

Böhringer, H., Matsushita, K., Churazov, E., Ikebe, Y., \& Chen, Y. 2002, A\&A, 382, 804
Churazov, E., Brüggen, M., Kaiser, C. R., Böhringer, H., \& Forman, W. 2001, ApJ, 554, 261

Cavaliere, A., \& Fusco-Femiano, R. 1976, A\&A, 49, 137

David, L. P., Slyz, A., Jones, C., et al. 1993, ApJ, 412, 479

De Grandi, S., \& Molendi, S. 1999, A\&A, 351, L45

Dickey, J. M., \& Lockman, F. J. 1990, ARA\&A, 28, 215

Ehle, M., Breitfellner, M., \& Dahlem, M., et al. 2001, XMM-Newton Users' Handbook, 14

Evrard, A. E., Metzler, C. A., \& Navarro, J. F. 1996, ApJ, 469, 494

Fabian, A. C., Arnaud, K. A., \& Nulsen, P. E. J., et al. 1985, MNRAS, 216, 923

Harris, D. E., \& Grindlay, J. E. 1979, MNRAS, 188, 25

Ikebe, Y., Reiprich, T. H., Böhringer, H., Tanaka, T., \& Kitayama, T. 2002, A\&A, 383, 773

Kaastra, J. S., Mewe, R., \& Nieuwenhuijzen, H. 1996, in UV and X-ray Spectroscopy of Astrophysical and Laboratory Plasmas, 411, ed. K. Yamashita, \& T. Watanabe (Tokyo: Univ. Ac. Press)

Loeb, A., \& Mao, S. 1994, ApJ, 435, L109

Lumb, D. 2001, XMM-Newton CCF Release Note, XMM-SOC-CAL$\mathrm{TN}-0016$

Machacek, M. E., Bautz, M. W., Canizares, C., \& Garmire, G. P. 2002, ApJ, 567, 188

Majerowicz, S., Neumann, D. M., \& Reiprich, T. H. 2002, A\&A, 394, 77

McNamara, B. R., Wise, M., \& Nulsen, P. E. J., et al. 2000, ApJ, 534, L135

McNamara, B. R., Wise, M., \& Nulsen, P. E. J., et al. 2001, ApJ, 562, L149

Miralda-Escudé, J., \& Babul, A. 1995, ApJ, 449, 18

Molendi, S., \& Pizzolato, F. 2001, ApJ, 560, 194

Mushotzky, R. F., \& Szymkowiak, A. E. 1988, Cooling Flows in Clusters and Galaxies, ed. A. C. Fabian

Pratt, G. W., \& Arnaud, M. 2002, A\&A, 394, 375

Sarazin, C. L., Baum, S. A., \& O’Dea, C. P. 1995, ApJ, 451, 125

Schmidt, R. W., Allen, S. W., \& Fabian, A. C. 2001, MNRAS, 327, 1057

Sun, M., Jones, C. Murray, S. S., Allen, S. W., Fabian, A. C., \& Edge, A. C. 2003, ApJ, 587, 619

Tamura, T., Kaastra, J. S., Peterson, J. R., et al. 2001, A\&A, 365, L87

Xue, S. J., \& Wu, X. P. 2002, ApJ, 576, 152

Wu, X. P. 1994, ApJ, 436, L115

Wu, X. P. 2000, MNRAS, 316, 299 